\title{
Driver seat comfort for level 3-4 autonomous vehicles
}

\author{
Neil Mansfield ${ }^{*}{ }^{1}$, Kartikeya Walia ${ }^{1,2}$ and Aditya Singh ${ }^{1,2}$ \\ ${ }^{1}$ Department of Engineering \\ School of Science and Technology \\ Nottingham Trent University \\ UK \\ ${ }^{2}$ University Institute of Engineering and Technology \\ Panjab University \\ Chandigarh \\ India \\ *corresponding author \\ neil.mansfield@ntu.ac.uk
}

Published in WORK, 2020

Please cite as:

Mansfield, N.J., Walia, K., and Singh, A. (2020) Driver seat comfort for level 3-4 autonomous vehicles. Work. 


\section{Abstract}

Background. Autonomous vehicles can be classified on a scale of automation from 0 to 5 , where level 0 corresponds to vehicles that have no automation to level 5 where the vehicle is fully autonomous and it is not possible for the human occupant to take control. At level 2, the driver needs to retain attention as they are in control of at least some systems. Level 3-4 vehicles are capable of full control but the human occupant might be required to, or desire to, intervene in some circumstances. This means that there could be extended periods of time where the driver is relaxed, but other periods of time when they need to drive.

Objective. The seat must therefore be designed to be comfortable in at least two different types of use case.

Methods. This driving simulator study compares the comfort experienced in a seat from a production hybrid vehicle whilst being used in a manual driving mode and in autonomous mode for a range of postures.

Results. It highlights how discomfort is worse for cases where the posture is non-optimal for the task. It also investigates the design of head and neckrests to mitigate neck discomfort, and shows that a well-designed neckrest is beneficial for drivers in autonomous mode.

Keywords: Autonomous vehicles, automated driving, seating, simulator study, backrest, comfort. 


\section{Introduction}

For the past century car seats have been developed primarily for drivers but with little emphasis on passengers or occupants in the rear seats. The classical driving posture involved the driver interacting with pedals and a steering wheel, and with the eyes continually looking at the road ahead or scanning mirrors, controls and displays. Seating postures are well defined and several standards have been produced such as SAE J287 [1] that gives guidance on driver hand control reach.

Technical innovation means that some level of automated control is becoming a standard feature in road cars. This could be a simple as anti-lock brakes, that are now mandated, or intelligent speed assistance, that will be a requirement for new cars in Europe from 2022. Autonomous vehicles are classified using six levels of automation as defined by SAE [2] (Table 1). From Level 3 and above the human 'driver' may move attention from the driving task. As appropriate for the technology and context, potentially the driver could release the steering wheel, desire to undertake another task (e.g. working, watching entertainment, sleeping), and sit in a different posture (Figure 1). Therefore the requirements for packaging the driver will be very different. Paddan et al. [3] showed that a reclined backrest at 157.5 degrees (i.e. almost reclined) was the most comfortable of a range of options when exposed to vertical whole-body vibration.

At Level 3 autonomy, the vehicle could request human intervention. Therefore the driver might be required to drive in a posture that is optimal for relaxing in the car, but not optimal for driving. At Level 2, drivers will be required to keep close attention on the road ahead forcing a posture that is able to maintain visual vigilance but is still relaxed. For Level $2 / 3$ autonomy, the human driver could be required to take control of the vehicle without 
opportunity to adjust the seat. This means that the vehicle must remain driveable by the human operator whilst in a 'relaxation' position.

\section{Table 1 about here}

\section{Figure 1 about here}

Whilst there have been several decades of research concerning optimization of the driving package, the driver posture and driver behavior, there has been limited consideration of the passenger. Passenger seats are configured to be almost identical to driver seats; the cost and logistics requirements for common parts across a product range that could be built into left- or right-hand-drive vehicles means that there is little scope for radically different seats on either side of the car. Furthermore requirements for safety performance during crash, and sustainability requirements in materials limit the scope of the design for the development team. The dual-mode of use in foreseeable autonomous cars means that drivers will switch between the role of driver and passenger. The driver's seat needs to be comfortable for both types of use-case.

This paper reports two studies. The first considered the effect of autonomous and manual driving on comfort in two postures that could be used at Level 3-4 autonomy; the second considered the effect of providing a neckrest to alleviate some issues observed in the first study.

The term 'autonomous' is used in this paper in preference to the term 'automated', as autonomous is now understood as 'self-driving car' by the general public [4]. It can be argued that 'automated' should be a preferred term especially at the lower SAE levels, as 
autonomous could suggest that the vehicle chooses its own destination. For consistency, autonomous is used here for all SAE levels.

\section{Methods}

A driving rig was developed for the study. The rig took overall dimensions from a production saloon car (Toyota Prius) and was custom built from aluminium extruded parts. Steering and seat position were adjustable such that they could be configured for any car model. A seat from a Toyota Prius was used in the driving rig. The visual display comprised four computer monitors each with a width of $630 \mathrm{~mm}$. One was placed directly in front of the driver and one placed at an angle of $55^{\circ}$ on either side of the front screen providing a nominally continuous field of view of $166^{\circ}$ interrupted by the two bezels. The vertical field of view from the primary screens was $21^{\circ}$. The driver sat at approximately $600 \mathrm{~mm}$ from the central screen, but this was affected by seat fore-aft adjustment. The fourth screen was positioned below the central screen and displayed a simulated instrument panel from the vehicle.

The software used in the simulation was Euro Truck Simulator 2 running on the Steam platform in Windows. This software provides a high-resolution simulation of European trunk roads including other road users, scenery, cities and variations in weather. The software was modified such that the vehicle in the simulation was a car rather than the default truck. This required modification of the graphics, the position of the driver, and the vehicle dynamics, handling and feedback. The software was also modified such that the vehicle would not require refuelling and that it would not be damaged if collisions occurred. 
Two sets of driving scenarios were programmed. Set 1 ('manual driving') comprised a journey in Great Britain that lasted 30 minutes and was guided using a software 'satnav' built into the simulation platform. The software included other road users and a mix of urban, rural and highway road types. Drivers needed to manually drive the simulation in this set. Set 2 ('autonomous mode') comprised a similar journey to that in Set 1 but the simulation ran in an autonomous mode such that the driver did not need to engage with the steering wheel or pedals in order for the journey to be completed. This scenario comprised a recording of the screens and audio made whilst an experienced user of the simulator drove the same route under the same setup.

Three different seating setups were used in the simulation. In the 'upright' position the seat backrest was set to $105^{\circ}$ representing a standard driving posture. In the 'reclined' posture the seat backrest was set to $127^{\circ}$ representing an extreme reclined driving posture, but similar to that used by passengers when relaxing, or in some racing cars and some military vehicles (Figure 2). The seat pan did not change in its inclination as the seat was from a production car without this feature. Backrest angles were selected in collaboration with the seat supplier to represent a standardised upright position for manual driving, as used in their internal testing protocols, and to represent an inclined posture at an extreme of practical adjustment that could be achieved in a car and driving is still possible. Other backrest angles could have been selected and might have been more appropriate for different cars.

In the 'reclined neckrest' posture drivers were provided with a neckrest designed to support the neck and head, optimised using FEA and piloted. The neckrest (Figure 3) was only used in conjunction with the reclined posture. Due to the extreme posture used in the study, it was not appropriate to use headrest designs that are found in production cars. 
The study was approved by NTU School of Science and Technology Human Ethics Committee, 17/18-60.

\subsection{Study 1 - Backrest angle and driving mode}

In Study 1, 7 male and 7 female participants were recruited from the staff and student population of Nottingham Trent University. All were experienced drivers. Participants were selected in order to represent a broad anthropometric range in stature. Other dimensions were not used for selection. Using American Adult anthropometric data participants were selected to be greater than 75 th percentile $(n=2$ male/female), between 25th and 75th percentile ( $n=3$ male/female), less than 25th percentile ( $n=2$ male/female) in stature.

Each participant visited the laboratory three times. The first visit to the laboratory was a familiarization and preparation session where participants were given instruction, training and information about the study. Participants were given an opportunity to learn how to drive the simulator and ask questions. During the other two visits participants completed four driving scenarios (two per lab visit), presented in a randomized Latinsquare order balanced to minimize order-effects. Between each scenario participants left the simulator and completed a 10 minute walk, which has previously been shown to be sufficient to give discomfort recovery [5]. 
The four driving scenarios comprised:

1. Autonomous mode in the upright posture

2. Autonomous mode in the reclined posture

3. Manual driving in the upright posture

4. Manual driving in the reclined posture

In the autonomous mode participants were asked to place their hands in their lap in a comfortable position. This was not externally controlled and they were free to reposition hands if desired. They did not hold any item in this condition. In the manual driving mode participants were free to grasp the steering wheel in any position, but to use two hands. In the reclined posture some participants were unable to reach the top of the steering wheel and therefore operated it from the bottom.

Participants were required to report their discomfort using the two-stage discomfort assessment protocol as previously used by Sammonds et al. [6]. Stage 1 of the protocol comprises a body part discomfort questionnaire where participants were asked to rate their discomfort on a 6 point scale adapted from ISO2631-1 [7]. Seven body regions were considered: lower back, upper back, neck, ankle, sitting bones, buttock area and edge of seat contact. These questions were designed to systematically prime the participant in order to generate reliable data from the main discomfort question which used an adapted Borg CR-100 scale. Participants reported their discomfort after 1, 15 and 29 minutes for each driving scenario. After completion of each scenario, participants were required to complete the NASA-TLX workload index [8] which uses weighted scores across 6 dimensions (mental demand, physical demand, temporal demand, perceived effort, perceived performance, frustration level). 
Participants were instructed to keep their eyes open in the autonomous conditions, and to maintain attention on the road. During debrief, no participant reported high levels of drowsiness; no participant was observed falling asleep.

\subsection{Study 2 - Effectiveness of neckrest at mitigating discomfort}

Study 2 also used 7 male and 7 female participants, but they were different individuals than used in Study 1. Participants were again selected to cover the anthropometric range. The protocol was similar to Study 1 except that five driving scenarios were used:

1. Manual driving in the upright posture

2. Manual driving in the reclined posture

3. Manual driving in the reclined posture with neckrest

4. Autonomous mode in the reclined posture

5. Autonomous mode in the reclined posture with neckrest

All participants had neck / head contact with the neckrest in scenarios 3 and 5 .

\section{Results}

There were no significant differences between discomfort scores for males and females for any of the conditions in either study. Therefore data were combined for further analysis. 


\subsection{Study 1 - Backrest angle and driving mode}

As expected discomfort increased with time for all conditions studied in Study 1 (Fig 4). In the autonomous mode only small differences were observed between the discomfort ratings in the upright and reclined postures. However, in the manual mode, discomfort was significantly greater for the reclined posture. These discomfort scores were elevated from a mean score in the 'moderate' discomfort range to 'high' discomfort for the manual, reclined.

Figure 4 about here

Individual body part discomfort scores were low for areas in contact with the seat pan cushion (Fig 5). Scores were highest in the neck region for the manual driving mode in the reclined posture. Increased discomfort was also apparent in the upper back. Verbatim comments also confirmed that the highest levels of discomfort occurred in the neck and upper back and this was attributed to the effort of holding the head in a position allowing for the driver to see the road ahead. Therefore, it was hypothesized that provision of a neckrest optimized for a reclined posture would mitigate the neck pain and improve the overall discomfort scores.

Figure 5 about here 


\subsection{Study 2 - Effectiveness of neckrest at mitigating discomfort}

In Study 2 the overall discomfort scores were not as great as those observed in Study 1. For those conditions that repeated those from Study 1, similar trends were observed with manual reclined being the most uncomfortable posture, and showing significantly more discomfort $(p<0.005$, t-test, 2-tailed; Fig 6$)$. In the reclined postures, when the neckrest was used, there was improvement in the discomfort scores for both manual and autonomous modes (Fig 7). Individual body part discomfort scores showed that there was no adverse effect on neck discomfort when in the reclined posture when a neckrest was used.

Figures 6 and 7 about here

The neckrest showed an immediate effect on discomfort (Figure 8). The reclined postures with no neckrest were more uncomfortable than all other postures for the duration of the trials. Neck discomfort increased over the 30 minutes period for all conditions.

Figure 8 about here

\section{Discussion}

Increases in discomfort with time were consistent across all conditions with higher levels of discomfort being reported at 29 minutes than after 1 minute in line with previous studies $[5,6]$. This increase was significant for $8 / 9$ conditions $(p<0.01$, t-test, 2 -tailed), but was not significant for manual driving in a reclined posture with neckrest $(p=0.057)$. All further statistical analyses are carried out for measurements made at 29 minutes. In the 
autonomous mode (study 1) backrest angle did not significantly affect the discomfort $(p=0.431)$. However, backrest angle affected discomfort for manual driving $(p<0.005)$ in both studies, where reclined driving was more uncomfortable. For the reclined backrest, the driving mode showed significant differences in discomfort in both studies $(p<0.05)$ where manual driving was more uncomfortable. In the upright posture there was no significant difference between the discomfort in manual or autonomous driving (study 1 , $\mathrm{p}=0.277$ ); similarly differences between discomfort ratings for manual and autonomous driving were not significant in the reclined posture with a neckrest (study $2, p=0.931$ ). The neckrest significantly improved the comfort for manual driving in a reclined posture $(p<0.005)$; although improvements were observed through the use of neckrest in the autonomous reclined mode, the difference did not reach significance $(p=0.051)$, although it should be noted that the autonomous reclined mode was significantly less uncomfortable than manual reclined.

The studies in this paper show that reclined postures can induce neck discomfort in both manual and autonomous modes. This might appear to contradict the results of Paddan et al. [3], but the previously published work used a flat seat, and importantly, the seat in the previous study included a padded headrest. The studies reported here demonstrate how a neckrest can be used to support the head to ensure that the experience of the user is optimized. The neckrest used in this study was a first iteration after initial design using anthropometry and finite element analysis to inform the contouring. The seat used in this study was from a production car and was not specifically designed for autonomous modes. Additional improvements are likely to be possible with further optimization of the seat design, the neckrest position and the backrest angle. This could include a full biomechanical analysis including consideration of neck loading for cases where there is a reclined posture with no neckrest. 
Perceived workload for the driving conditions in this study showed that there was an increase in NASA-TLX score, indicating a higher workload, when in the reclined posture for both studies (study 1: 44.5 vs. $54.2, p<0.05$; study 2: 46.9 vs. $56.6, p<0.05$ ). The introduction of the neckrest improved the perceived workload (study 2: 43.1, $\mathrm{p}<0.005$ ). This indicates that the adverse effects of the reclined posture on perceived human performance can also be mitigated through the introduction of a neckrest.

This study did not simulate whole-body vibration, lateral (cornering) forces or longitudinal loading (acceleration / braking). The seat and neckrest used may also require additional work to optimise for these loads in order to maintain torso and head stability in the reclined posture.

It is concluded that reclined postures can increase the discomfort experienced by drivers when driving manually or if they need attend to the road ahead when in autonomous mode. The introduction of a neckrest improves comfort sensations and also improves the perceived workload. Therefore automotive seat designs for autonomous vehicles need a new set of design criteria in order to maximize the sensation and wellbeing of the driver.

Acknowledgments The research work reported here was made possible by the support of Bridgestone Corporation, Japan.

\section{References}

1. Society for Automotive Engineers. SAE J287. Driver Hand Control Reach. 2016. 
2. Society for Automotive Engineers. SAE J3016. Taxonomy and Definitions for Terms Related to Driving Automation Systems for On-Road Motor Vehicles. 2018.

3. Paddan, G.S., Mansfield, N.J., Arrowsmith, C.I., Rimell, A.N., King, S.K. and Holmes, S.R., 2012. The influence of seat backrest angle on perceived discomfort during exposure to vertical whole-body vibration. Ergonomics, 55(8), pp.923-936.

4. Wood, S. P.; Chang, J.; Healy, T.; Wood, J. "The potential regulatory challenges of increasingly autonomous motor vehicles". 52nd Santa Clara Law Review. 4 (9): 1423-1502.

5. Sammonds, G.M., Mansfield, N.J. and Fray, M., 2017. Improving long term driving comfort by taking breaks-How break activity affects effectiveness. Applied Ergonomics, 65, pp.81-89.

6. Sammonds, G.M., Fray, M. and Mansfield, N.J., 2017. Effect of long term driving on driver discomfort and its relationship with seat fidgets and movements (SFMs). Applied Ergonomics, 58, pp.119-127.

7. International Organization for Standardization 1997, Mechanical vibration and shock-Evaluation of human exposure to whole-body vibration - Part 1: General requirements, ISO 2631-1.

8. Hart, S. G. and Staveland, L. E. 1988. "Development of a NASA-TLX (Task Load Index): results of empirical and theoretical research". In Human mental workload, Edited by: Hancock, P. A. and Meshkati, N. Amsterdam: Elsevier Science Publishers B.V. 
Table 1. Levels of Automation as defined by the Society of Automotive Engineers [2].

\begin{tabular}{|c|c|}
\hline Level & Description \\
\hline Level 0 No automation: & $\begin{array}{l}\text { No direct vehicle control, but warning systems may be } \\
\text { present (e.g. parking sensors). }\end{array}$ \\
\hline $\begin{array}{l}\text { Level } 1 \text { Driver } \\
\text { assistance: }\end{array}$ & $\begin{array}{l}\text { Automated speed (cruise) control, lateral (lane keeping) } \\
\text { control, and parking assistance. }\end{array}$ \\
\hline $\begin{array}{l}\text { Level } 2 \text { Partial } \\
\text { automation: }\end{array}$ & $\begin{array}{l}\text { System can take full control of lateral and longitudinal vehicle } \\
\text { motion, but human supervisor is necessary to re-take control } \\
\text { at any time. }\end{array}$ \\
\hline $\begin{array}{l}\text { Level } 3 \text { Conditional } \\
\text { automation: }\end{array}$ & $\begin{array}{l}\text { The driver can move their attention from the driving task in } \\
\text { well-controlled environments (e.g. highways), but is needed } \\
\text { to manually drive the car in complex scenarios. The car can } \\
\text { take decisions on whether to overtake and can request a } \\
\text { rapid return to human control. }\end{array}$ \\
\hline $\begin{array}{l}\text { Level } 4 \text { High } \\
\text { automation: }\end{array}$ & $\begin{array}{l}\text { The car can drive itself in almost all circumstances. Human } \\
\text { control may be needed if systems fail (e.g. in poor weather) } \\
\text { but the car can safely proceed if the driver is unable to take } \\
\text { control. Human control may be possible at the human's } \\
\text { request. }\end{array}$ \\
\hline Level 5 Full automation: & $\begin{array}{l}\text { There is no possibility for the human operator to physically } \\
\text { drive the car. The human occupant is effectively a } \\
\text { passenger. }\end{array}$ \\
\hline
\end{tabular}




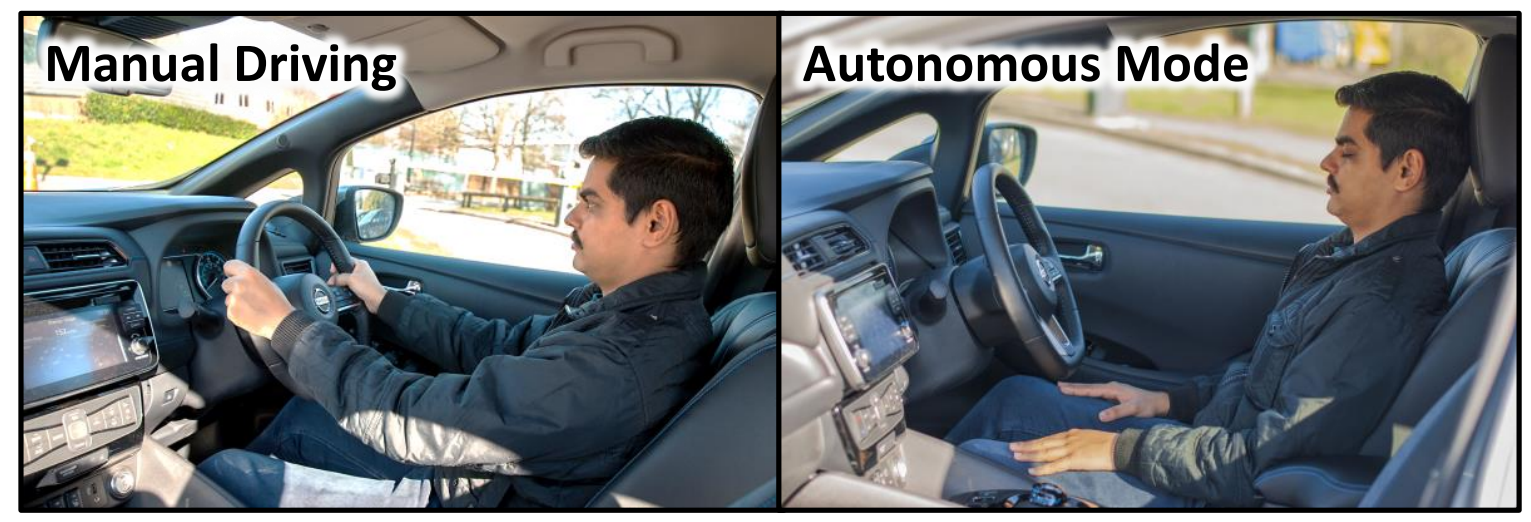

Fig. 1. Manual driving assumes hand, feet and head position in a standard posture. In autonomous mode, these assumptions may no longer hold. 

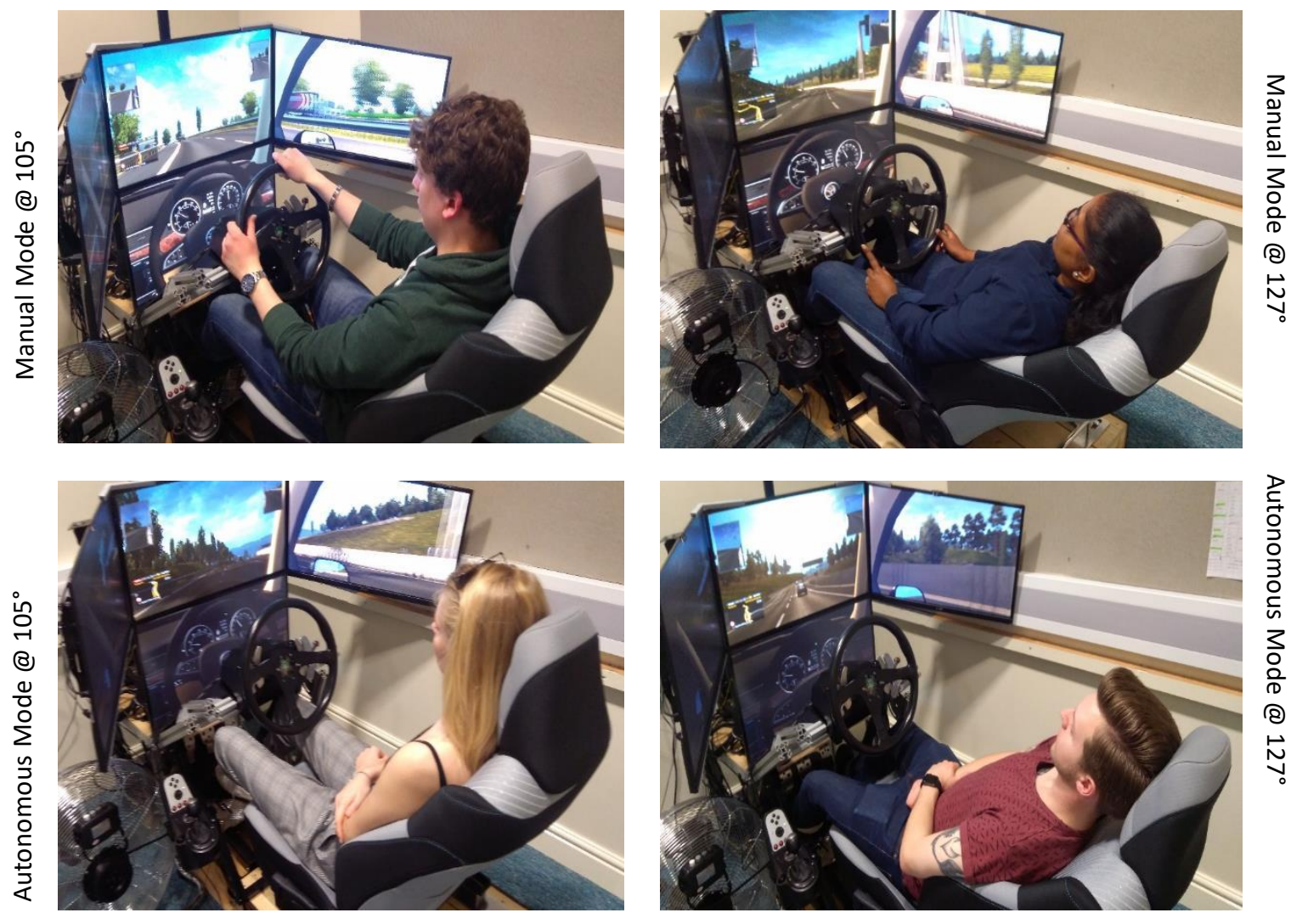

Fig. 2. Lab setup showing manual and autonomous modes in the two driving positions. 

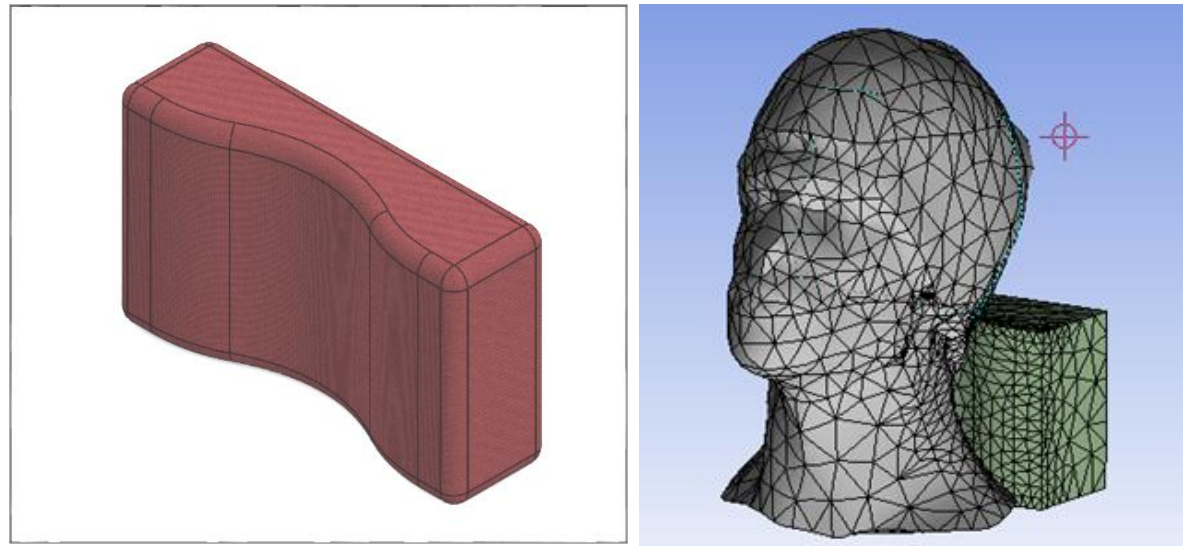

Fig. 3. CAD model of neckrest used in the reclined driving positions in Study 2. 


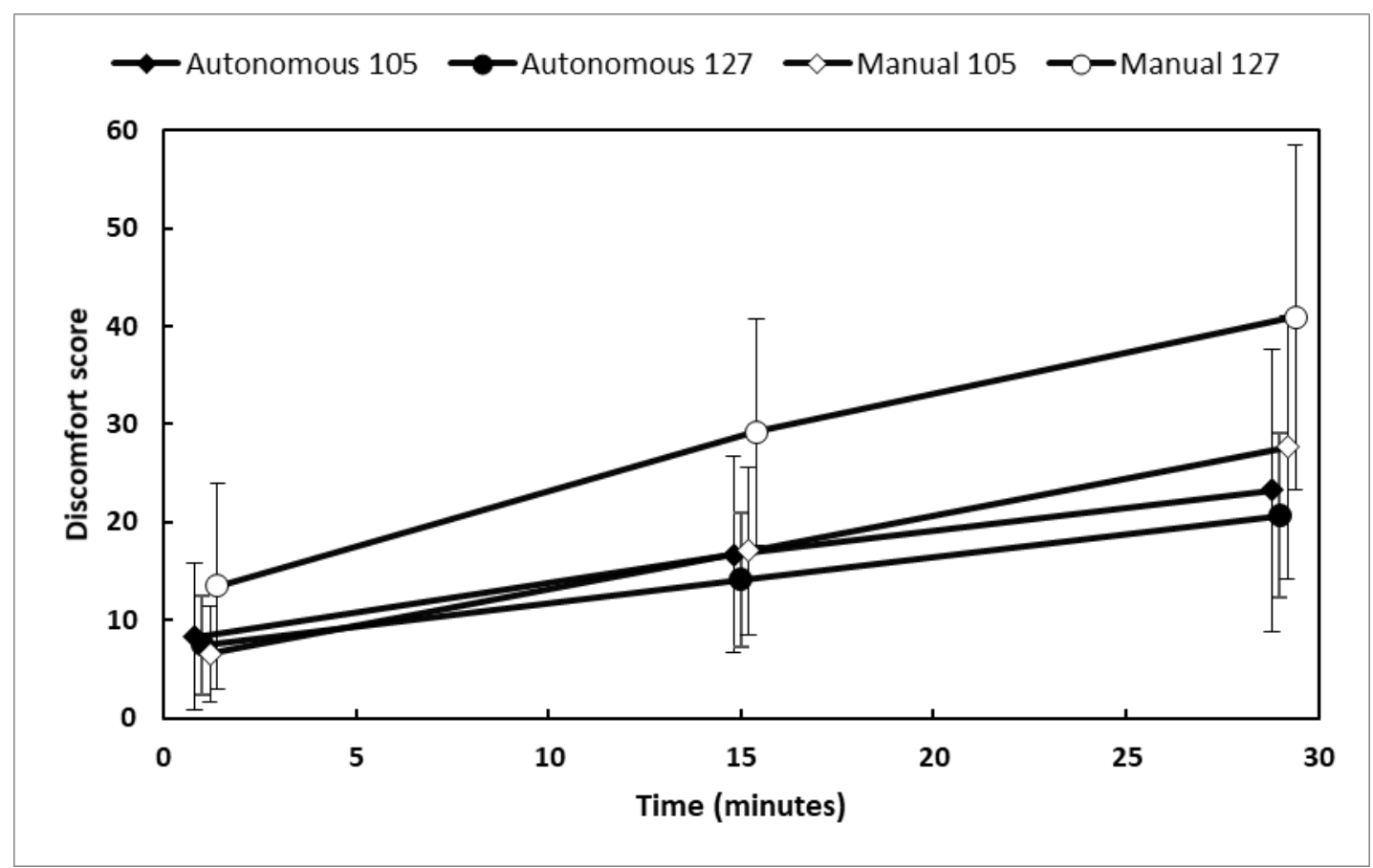

Fig. 4. Overall discomfort ratings for study one showing the effect of driving mode, posture, and time. Data show mean and standard deviation. Jitter on x-axis added for clarity; measurements were made at 1,15 and 29 minutes. 


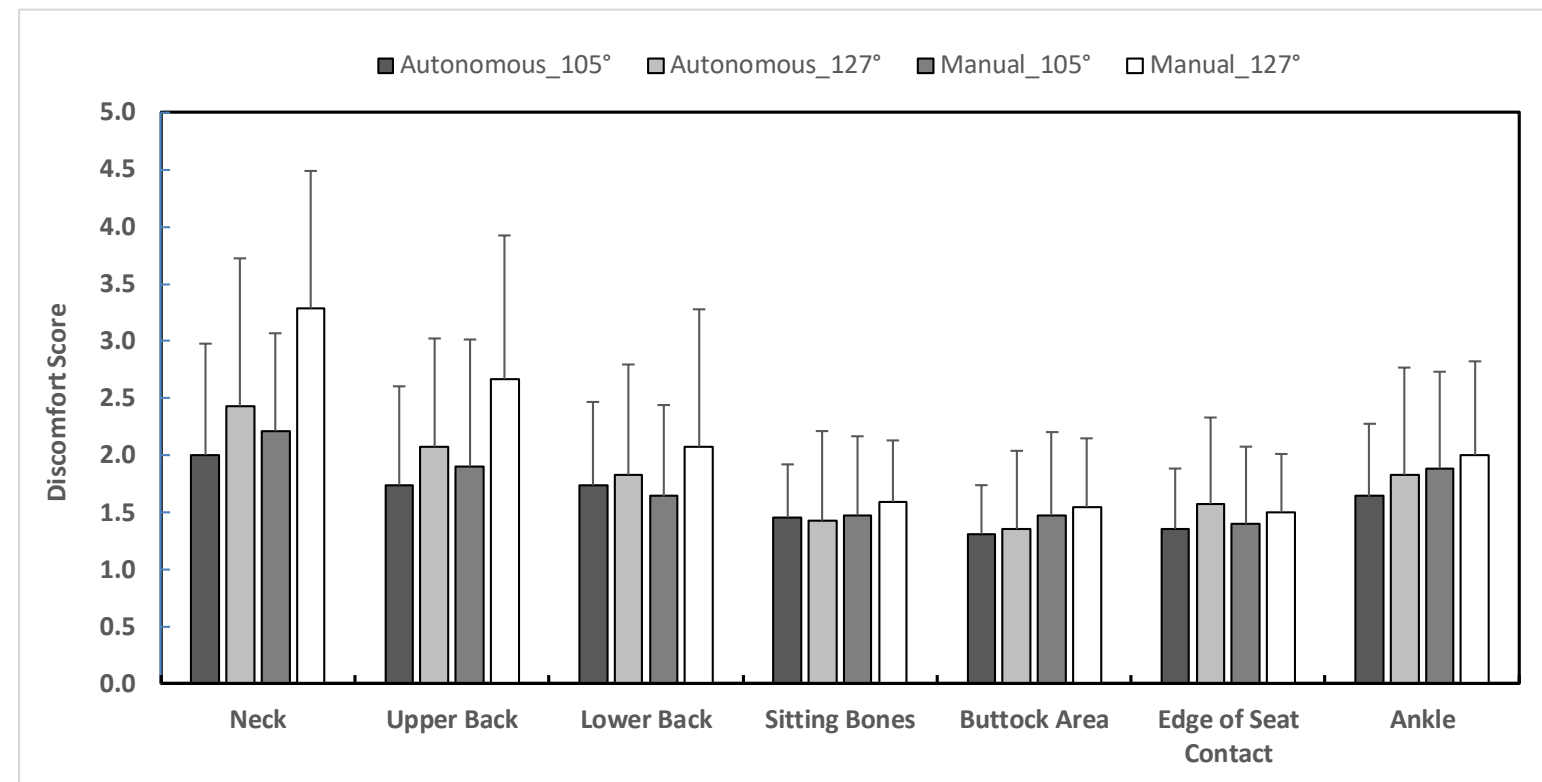

Fig. 5. Individual body part discomfort for Study 1 measured at 29 minutes. Data show mean and standard deviation. 


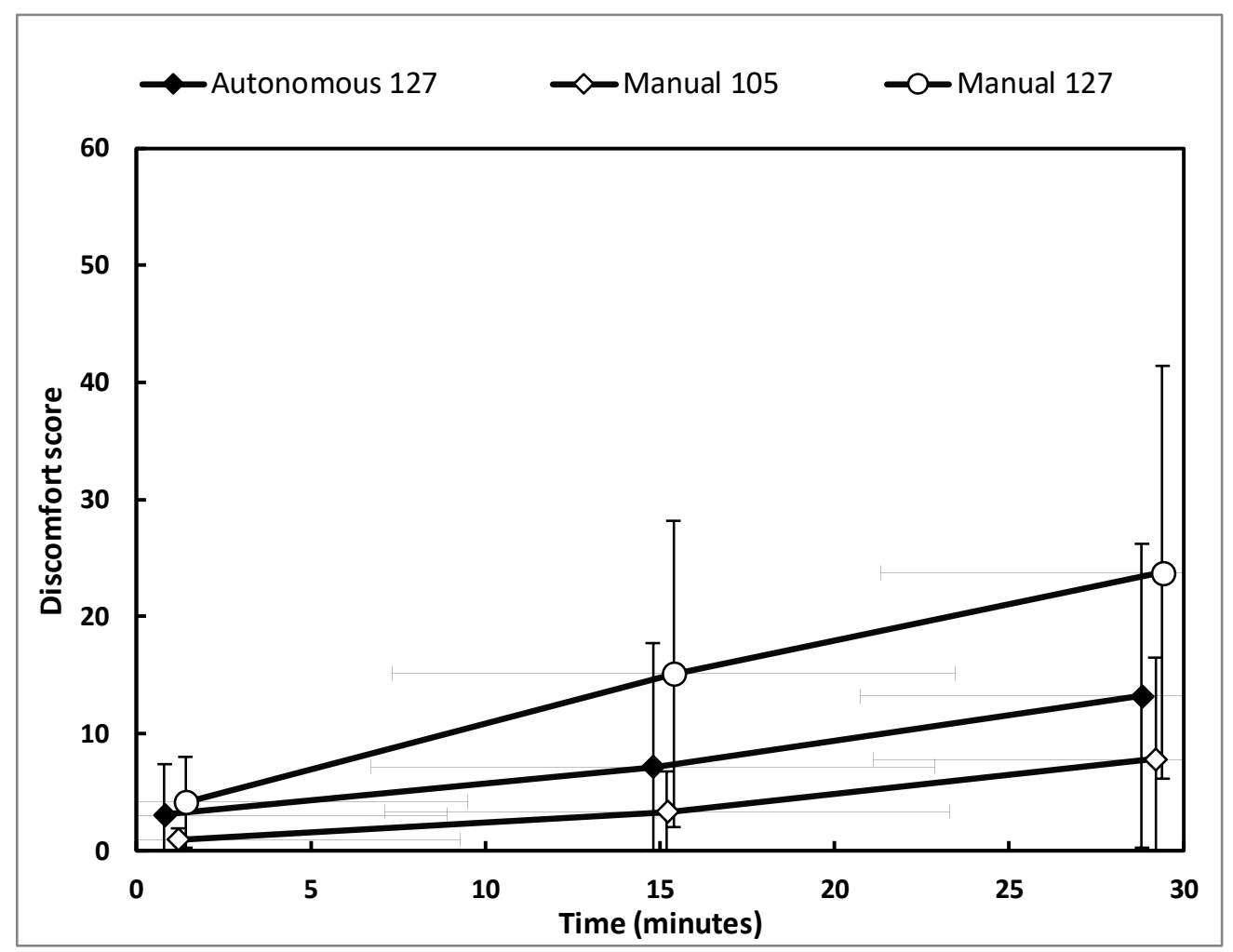

Fig. 6. Overall discomfort ratings for study two showing the effect of driving mode, posture, and time for conditions with no neckrest. Data show mean and standard deviation. Jitter on x-axis added for clarity; measurements were made at 1, 15 and 29 minutes. 

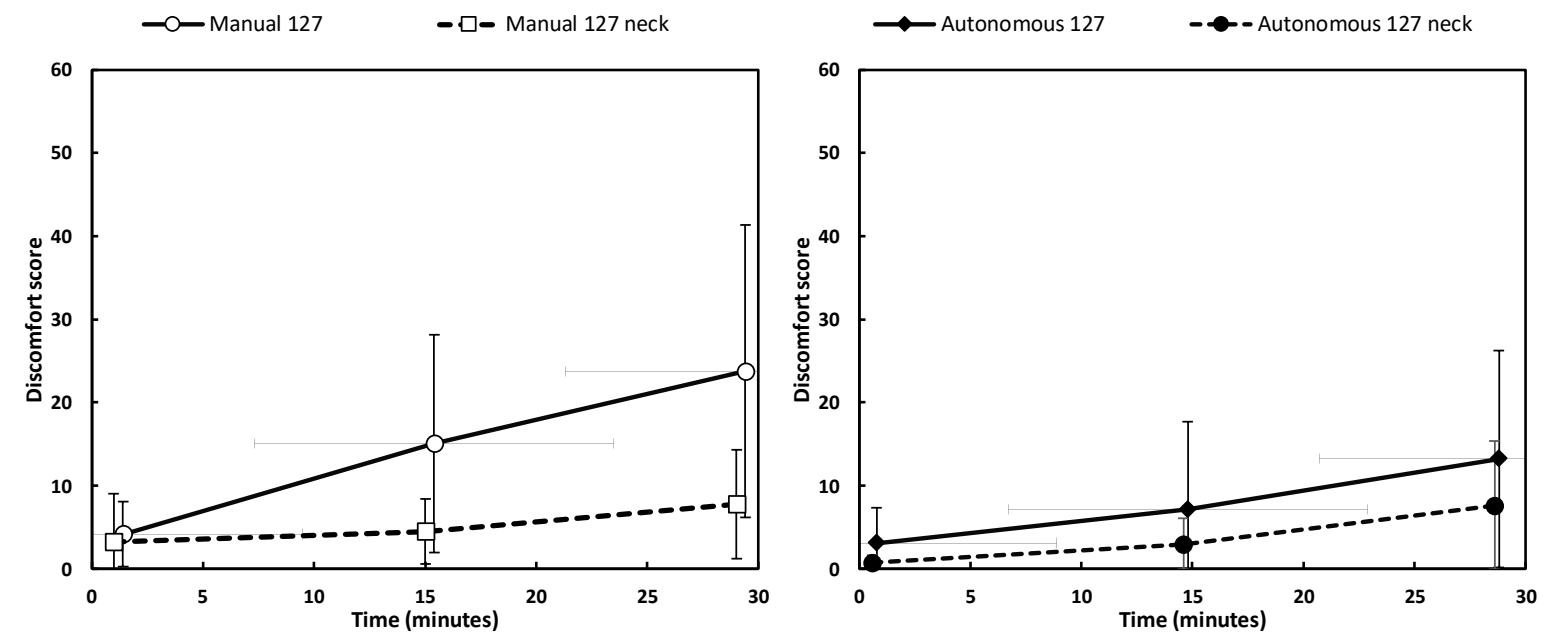

Fig. 7. Overall discomfort measured for manual and autonomous driving with and without neckrest in Study 2. Data show mean and standard deviation. Jitter on x-axis added for clarity; measurements were made at 1,15 and 29 minutes. 


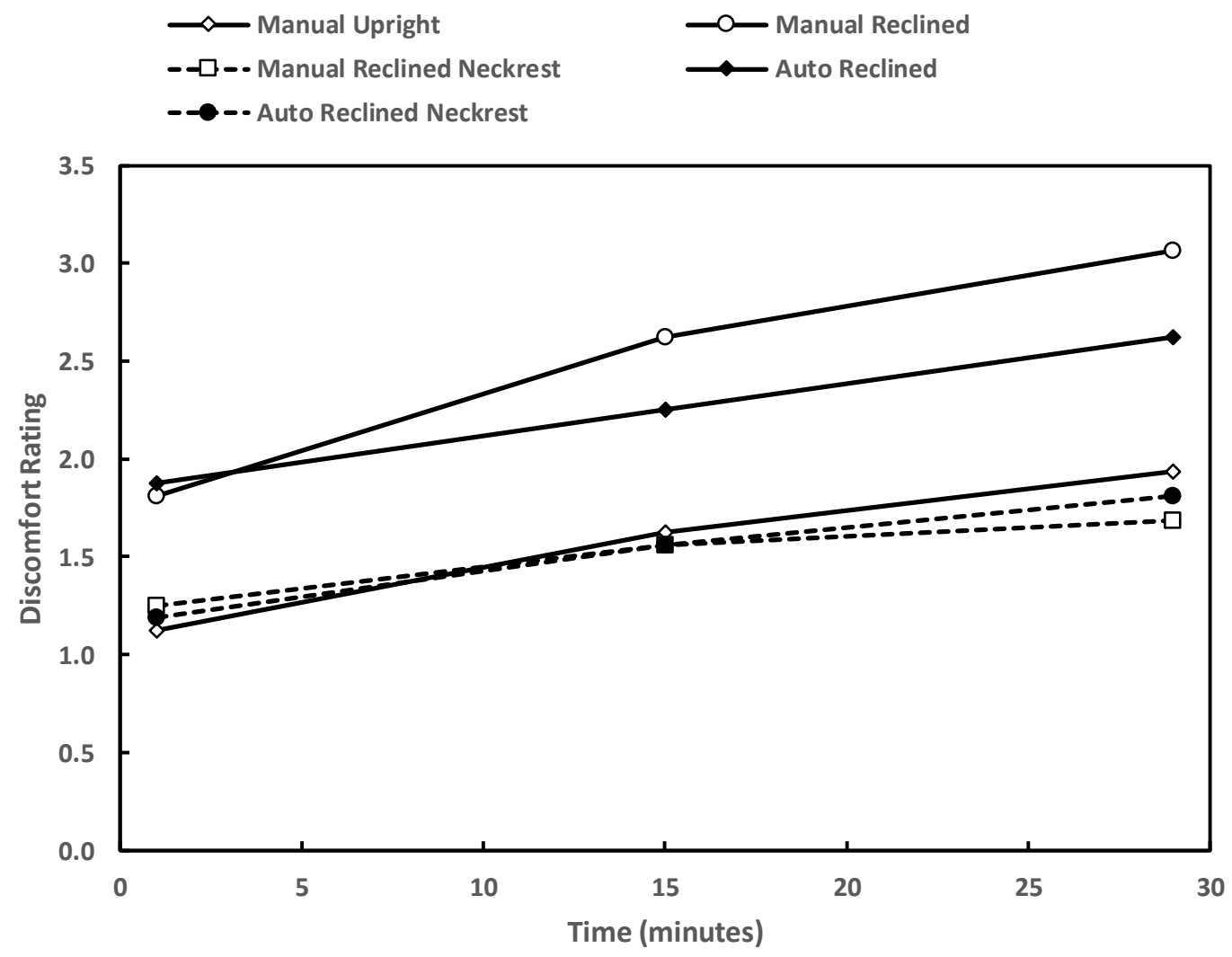

Fig. 8. Mean neck discomfort measured for manual and autonomous driving with and without neckrest in upright and reclined postures. Effect of time on neck discomfort. 